\title{
Real-Time Order Tracking of Gear Mesh Vibration in High Speed Planetary Gearboxes
}

\author{
Daniel Fritz Plöger ${ }^{1, \star}$, Philipp Zech ${ }^{1}$, and Stephan Rinderknecht ${ }^{1}$, \\ ${ }^{1}$ Mechatronic Systems in Mechanical Engineering, Technische Universität Darmstadt
}

\begin{abstract}
Possible approaches to real-time order tracking are discussed. Two methods for real-time order tracking are developed and validated experimentally for the entire audible spectrum. An adaptive heterodyne filter bank is compared to a direct integral transform. The performance of both methods is adequate for usage in an active vibration control (AVC) algorithm. Vold-Kalman filters are not suitable for AVC. The vibration data of three different planetary gearboxes is analyzed using order tracking. While some of the existing research could be reproduced, the data contradicts statements made by several authors. Lastly, the architecture of a novel AVC algorithm is sketched out.
\end{abstract}

\section{Introduction}

The vibration of planetary gearboxes is highly modulated. An explanation for this has been reported first by McFadden and Smith [1]. For an accurate analysis of the vibration a technique called order tracking has to be employed. Order tracking decomposes the vibration signal into its individual spectral components, often referred to as orders. It is mostly performed off-line using recorded vibration data. Borghesani et al. [2] explain how this can be performed using state of the art methods. They categorize the known approaches into three classes: "the resampling methods, the Kalman filter based methods and the transform based methods". For off-line analysis, resampling remains a popular choice because of its accuracy and robustness.

The goal of this paper is to extend order tracking to real-time and to fast running planetary gearboxes. The spectral components of the vibration signal are extracted at the same time they occur. While real-time order tracking can still be used for off-line analysis, it may also be used for on-line condition monitoring or active vibration control (AVC). Here, the focus will be on AVC.

The class of resampling methods will not be investigated in this paper, because these inherently rely on recorded data. Instead, a transform based concept will be developed, leading to two different methods. The VoldKalman filter (VKF) will also be discussed briefly. The obtained methods will be implemented on a test rig and a brief analysis of the experimentally acquired gearbox vibration will be given. Lastly, an AVC algorithm using real-time order tracking will be sketched out. However, an implementation of AVC is not within the scope of this paper.

\section{Experimental Set-Up}

The experimental set-up is depicted in Figure 1 and consists of an asynchronous motor (1) that drives the sun gear of the planetary gearbox (3) and an eddy current brake (5) that is connected to the planet carrier. Metal bellow couplings (2) and (4) connect the shafts of motor, eddy current brake and gearbox. Using this setup the gearbox can be operated at input speeds up to $10000 \mathrm{~min}^{-1}$ and output torques up to $40 \mathrm{Nm}$. The gearbox mount is supported by two triaxial force sensors (6). Figure 1 shows the coordinate systems for both sides of the mount. Furthermore the gearbox mount is equipped with eight accelerometers (7). The experimental setup allows measuring the gear mesh forces that are transmitted through the gearbox mount (8) into the surrounding structure. This work focuses on the data of the accelerometer on the right side of the gearbox mount in $x_{1}$ direction to investigate the online order-tracking approaches. The experimental setup enables measurement of run-ups as well as stationary operation. A speedgoat performance real-time target machine featuring an Intel Core i7 $3770 \mathrm{~K} 3.5 \mathrm{GHz}$ quadcore CPU allows for a frequency of $80 \mathrm{kHz}$ in closed loop operation. The piezoelectric accelerometers are of type MMF KS78B.10 and their signal is fed into a MMF M208B measurement amplifier featuring a low-pass filter with cut-off frequency of $30 \mathrm{kHz}$. Three commercially available planetary gearboxes are selected for the investigation as presented in Table 1.

\section{Modeling Gear Mesh Vibration}

It is known from existing research [3] that the vibration of planetary gearboxes may be modeled accurately as a Fourier series. Using this approach, a vibration model has been presented by the authors [4]. This paper will extend its application to real time. On behalf of briefness, only a

\footnotetext{
^e-mail: ploeger@ims.tu-darmstadt.de
} 
Table 1. Selected planetary gearboxes.

\begin{tabular}{llll}
\hline Gearbox & A & B & C \\
\hline Gear & spur & spur & helical \\
$Z$ & 84 & 84 & 108 \\
Ratio & 3 & 3 & 3 \\
Outer diameter & $60 \mathrm{~mm}$ & $40 \mathrm{~mm}$ & $68 \mathrm{~mm}$ \\
Mass & $0.9 \mathrm{~kg}$ & $0.35 \mathrm{~kg}$ & $1.9 \mathrm{~kg}$ \\
\hline
\end{tabular}

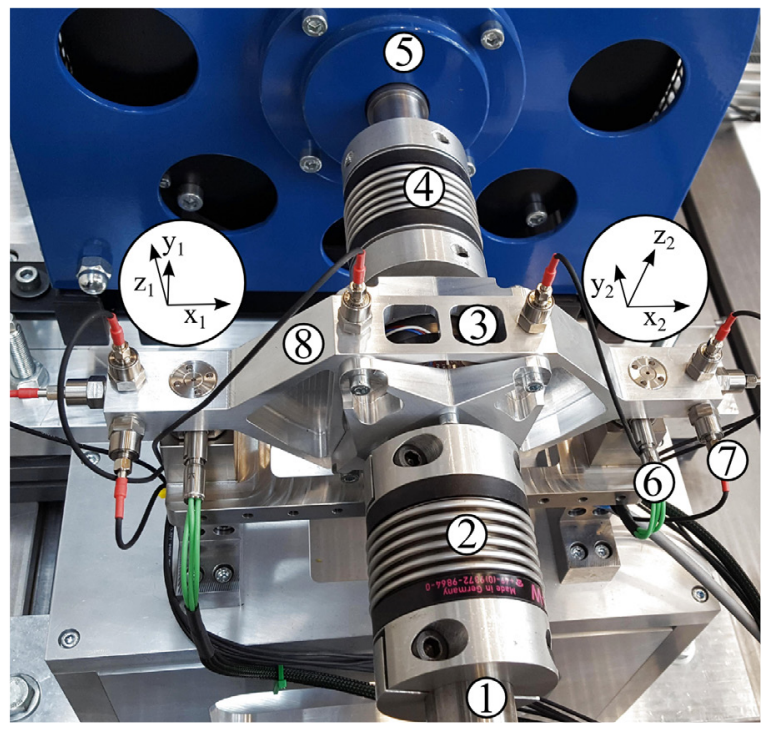

Figure 1. Set-up: Motor shaft (1), metal bellow couplings $(2,4)$, planetary gearbox (3) within gearbox mount (8), eddy current brake (5), triaxial force sensors (6) and accelerometers (7).

rough outline on the vibration model will be discussed. For a detailed derivation refer to [4]. In this paper planetary gearboxes with a fixed ring gear will be discussed.

The vibration of a planetary gearbox can be expressed as a modulated signal. This has been first recognized by McFadden and Smith [1]. The fundamental frequency corresponds to the nominal gear mesh frequency $\omega_{0}$, which is defined by the angular velocity of the planet carrier $\dot{\varphi}$ and the number of teeth on the ring gear $Z$.

$$
\omega_{0}=Z \dot{\varphi}
$$

If the angular velocity is constant, the modulation will be periodic w. r.t. time. In transient operation the modulation $A$ can be modeled periodic w.r.t. the carrier angle $\varphi$, if the angular acceleration is not too large. In this model, a complex modulation $A$ will be used. Two counter rotating phasors are introduced in order to constrain the signal $v$ to real values.

$$
v=\frac{1}{2} A(\varphi) e^{i \omega_{0} t}+\frac{1}{2} \bar{A}(\varphi) e^{-i \omega_{0} t}
$$

The signal $v$ may represent any observable vibration, such as a force, acceleration, displacement or similar. Because the modulation $A$ is periodic w.r.t. the angle $\varphi$ of the planet carrier it may be expressed as a Fourier series. In anticipation of the next step the index of the series has already been shifted by the number of teeth $\mathrm{Z}$.

$$
A(\varphi)=\sum_{k=-\infty}^{\infty} c_{k-Z} e^{i k \varphi}
$$

The shift is only a change in notation from a mathematical standpoint. However, the shifted index $k$ is closely connected to the properties of the vibration signal. This will be validated later in Section 7. In summary it means that the most significant vibration occurs at frequencies close to the nominal gear mesh frequency $\omega_{0}$. It also means that the spectral components are spaced evenly in the frequency domain at a distance corresponding to the frequency $\dot{\varphi}$ at which the planet carrier rotates. Thus, the index $k$ uniquely identifies a spectral component. Now (3) is inserted into (2) and (1) is applied to the result.

$$
v=\frac{1}{2}\left[\sum_{k=-\infty}^{\infty} c_{k} e^{i k \varphi}+\sum_{k=-\infty}^{\infty} \bar{c}_{k} e^{-i k \varphi}\right]
$$

Without loss of generality it may be assumed that the coefficients $c$ are complex conjugate pairs.

$$
c_{k}=\left\{\begin{array}{ll}
a_{0} & \text { for } k=0 \\
\frac{1}{2}\left(a_{k}-i b_{k}\right) & \text { for } k>0 \\
\frac{1}{2}\left(a_{-k}+i b_{-k}\right) & \text { for } k<0
\end{array} .\right.
$$

Therefore (4) reduces to

$$
v=\sum_{k=-\infty}^{\infty} c_{k} e^{i k \varphi}=\frac{a_{0}}{2}+\sum_{k=1}^{\infty}\left(a_{k} \cos k \varphi+b_{k} \sin k \varphi\right) .
$$

The expressions $\cos k \varphi$ and $\sin k \varphi$ may be interpreted as reference oscillators.

This very basic model of the vibration signals may serve different purposes. It is a useful analytical tool because it reduces substantial amounts of data to a set of coefficients. It compares favorably to the use of the FFT, which does not reduce the amount of data without averaging of the spectrum. In contrast to FFT based approaches it also utilizes the sparsity of the vibration signal in the frequency domain. The FFT of steady-state gearbox vibration is zero at many frequencies. In addition, approximately $80 \%$ of the nonzero spectral lines are only nonzero because of spectral leakage. The model (6) presumes this structure inherently and does not need to express it explicitly by yielding vanishing spectral components at certain frequencies. Also it performs acceptably in the case of non-constant angular velocity. Here FFT based approaches need to re-sample the measured data as explained in [2].

If used for analysis, the task is to compute the coefficients $c_{k}$ from measured data. However, the model is not limited to analysis. Because it describes the vibration of planetary gearboxes precisely and efficiently, it is suited as the foundation from which active vibration control can be derived. This will be sketched out in Section 8 . The intermediate goal is to arrive at a real-time capable estimation of $c_{k}$, where the estimated coefficients are updated on-line at a rate of $80 \mathrm{kHz}$, observing the entire audible 


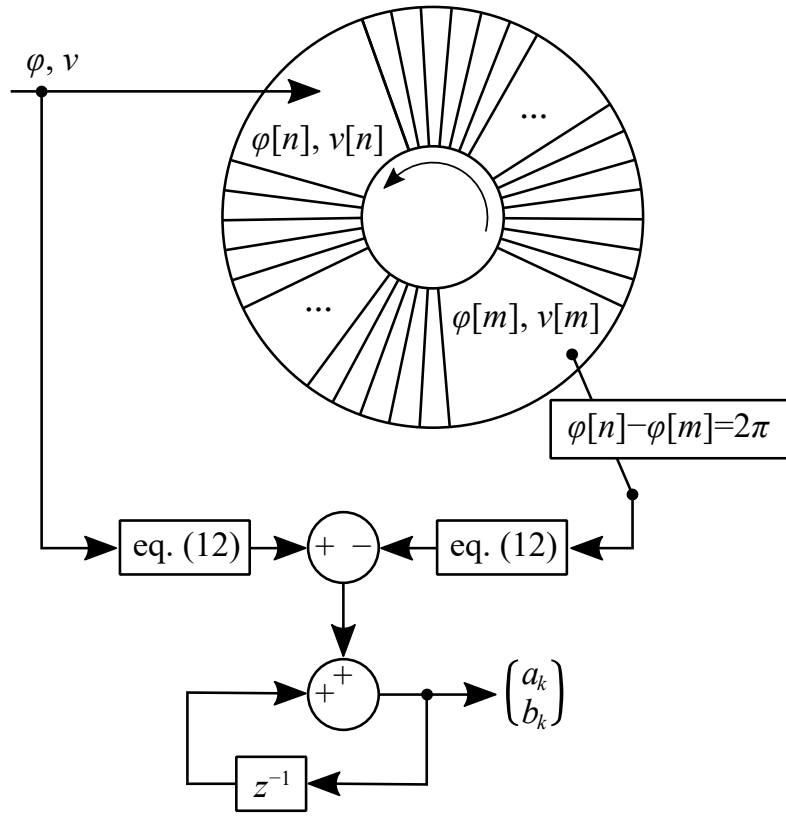

Figure 2. Ring buffer structure.

range. In the following sections, three possible approaches will be discussed: First, the coefficients may be calculated by integral transform. Second, a heterodyne filter bank may be employed. Third, a Vold-Kalman filter can be used.

\section{Integral Transform}

From the infinite series of (6) any coefficient $c_{k}$ may be computed by solving

$$
a_{k}=\frac{1}{\pi} \int_{0}^{2 \pi} v(\varphi) \cos k \varphi d \varphi
$$

and the analogous Equation for $b_{k}$. Because the only difference between $a_{k}$ and $b_{k}$ is the use of sin instead of cos, the derivation of $b_{k}$ is mostly omitted from this study. More details may be found in [4]. For a numerical computation the continuous time signals have to be substituted by their discrete time approximations

$$
\begin{aligned}
& a_{k} \approx \frac{1}{2 \pi} \sum_{l=m}^{n} v[l](\varphi[l+1]-\varphi[l-1]) \cos k \varphi[l] \\
& b_{k} \approx \frac{1}{2 \pi} \sum_{l=m}^{n} v[l](\varphi[l+1]-\varphi[l-1]) \sin k \varphi[l]
\end{aligned}
$$

where the start index $m$ and the stop index $n$ have to satisfy the limits of the original integral, such that

$$
\varphi[n]-\varphi[m] \approx 2 \pi
$$

The method was originally used for off-line analysis in [4]. This approach may be adapted to real-time. In this realtime context the index $n$ denotes the current step. From (10) it follows that the index $m[n]$ is a function of $n$, because at any time $n$ there needs to be one full turn of the planet carrier between $m$ and $n$.
The sum in Eq. (8) may be split up in its individual summands.

$$
\begin{aligned}
a_{k}[n] & =\sum_{l=m[n]}^{n} \Delta a_{k}[l] \\
\Delta a_{k}[l] & =\frac{1}{2 \pi} v[l](\varphi[l+1]-\varphi[l-1]) \cos k \varphi[l] \\
a_{k}[n] & =a_{k}[n-1]+\Delta a_{k}[n]-\sum_{l=m[n-1]}^{m[n]-1} \Delta a_{k}[l]
\end{aligned}
$$

During stationary operation in every time step there will be one summand added and one will be subtracted. If the gearbox speeds up, more than one summand needs to be removed. Conversely at time steps where the gearbox slows down there it is possible that no summand needs to be subtracted.

In a practical implementation this will be realized using a circular buffer memory as visualized in Figure 2. This buffer is able to store a certain amount of past data. Its length is adjusted to the lowest angular velocity of the planet carrier at which the algorithm is to be executed.

Numerical stability has to be considered. In fixed point arithmetic, the summands added can be removed exactly by subtraction. In a floating point arithmetic this is not always true, as the exponent might change after addition. If single precision floating point arithmetic is used, the coefficients may have to be reset in regular intervals, which can be done without loss of information. Using double precision, the round-off error can safely be ignored. Here, double precision arithmetic will be used.

\section{Heterodyne filtering}

The heterodyne principle moves the contents of a time domain signal in the frequency domain. This technique has been known for more than a century. For the historical context see [5] or [6]. The principle has been used in radio applications since. It also may be employed to create digital adaptive band pass filters as discussed in [7].

The heterodyne principle has been applied to planetary gearboxes before. [8]. However, it has been employed to shift high frequency acoustic emission signals below the Nyquist frequency of the DAQ hardware. This paper uses the heterodyne principle for an entirely different purpose. Here, instead of a single heterodyne unit, a filter bank consisting of several units is used to decompose the signal in its spectral components. The filter bank needs to be adaptive because the frequencies at which the components are located depend on the angular velocity of the planet carrier.

Figure 3 shows how an adaptive band pass filter may be realized. From the signal $v$ the $k$-th spectral component will be extracted. The instantaneous angle $\varphi$ of the planet carrier is fed into the reference oscillator. Here, the filter is split into a real path (cosine) and an imaginary path (sine). All operations are performed equally on both paths. The mixer multiplies the signal $v$ by the output of the reference oscillator. 


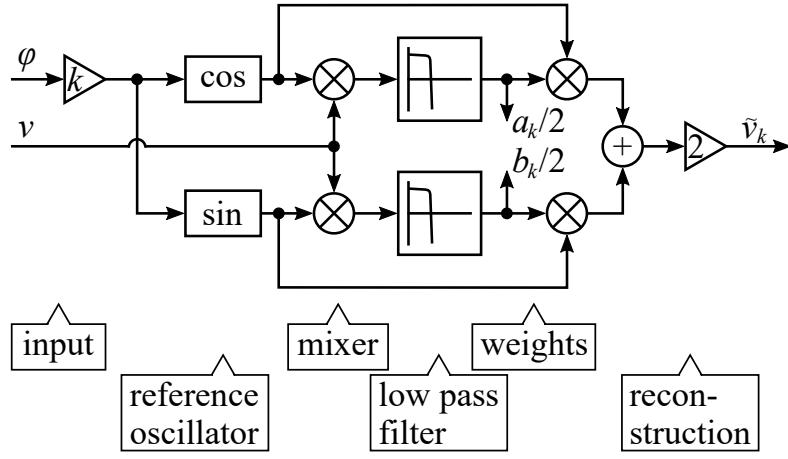

Figure 3. Adaptive heterodyne band pass filter.

This mixing is the key step in the heterodyne principle. It shifts the frequency of the $k$-th spectral component to the base band. Because of this, an ordinary low pass filter can be used to remove all other spectral components. The design of the filter determines the actual performance of the heterodyne unit. It will be discussed in detail later. Because the frequency of the spectral component of interest has been shifted to DC, the two low pass filters extract real and imaginary part of the Fourier coefficient $c_{k}$. Multiplication of these weights by the reference oscillation yields a reconstruction of the time domain signal.

Of course, this arrangement only extracts one spectral component $\tilde{v}_{k}$ from $v$.

$$
\tilde{v}_{k}=a_{k} \cos k \varphi+b_{k} \sin k \varphi
$$

In order to extract multiple components, a parallel array of identical units has to be employed. This is known as a filter bank. It is able to extract any number of Fourier coefficients concurrently. In turn, using the extracted coefficients and the reference oscillators, the original signal $v$ may be reconstructed. The quality of a filter design may be judged by the reconstruction error

$$
\varepsilon=v-\sum_{k=0}^{N} \tilde{v}_{k}
$$

where $N$ is the Number of spectral components present in the signal.

However if the filter exhibits a phase of $\pm \pi / 2$ in the stop band, the reconstruction error will be a superficial measure of the performance of the filter design. This can be most easily explained using the experimental data of Section 7. Consider the spectral component of the highest magnitude. For gearbox A this is $k=83$ at most operating conditions. If the planet carrier turns at $\dot{\varphi} / 2 \pi=3000 \mathrm{~min}^{-1}$, the distance between the spectral components will amount to $50 \mathrm{~Hz}$. Heterodyne unit number 82 will shift spectral component to $50 \mathrm{~Hz}$ with a phase of $-\pi / 2$ and an attenuation specific to the filter design. Unit 84 will also shift it to $50 \mathrm{~Hz}$, but with a phase of $\pi / 2$ and the same amount of attenuation. In Equation (15) this will cancel out. While the reconstruction is successful, the decomposition is not.

Any low pass filter may be used in the heterodyne unit, but the performance relies on a good choice. Generally FIR filters are not investigated in this paper. Compared to IIR filters they require a much higher order to achieve equal performance. At a desired cutoff frequency of less than 1/1000 of the Nyquist frequency FIR filters are computationally too expensive. A FIR design would need a length of more than 5000 to achieve acceptable performance.

A Chebyshev type II design has been chosen for the low pass filter. If the order of this filter is odd, the phase will be $-\pi / 2$ in the stop band. Only for even orders numerical optimization of the reconstruction error is sensible. Because the filter will be implemented as a biquad structure, an order of 3 would offer no advantage over an order of 4 regarding the computational effort. Orders 1 or 2 cannot yield valid filter designs. Therefore, an order of 4 has been chosen. Generally, no improvements could be achieved by higher filter orders.

The filter may be characterized by the parameters stopband frequency $f_{\text {stop }}$ and stopband attenuation. They determine the selectivity of the filter. This means the ability to extract the spectral component of interest and to reject any other component. As explained in Section 3, the distance between the spectral components corresponds to the rate at which the planet carrier rotates. Therefore the stopband frequency $f_{\text {stop }}$ must never exceed this.

$$
0<f_{\text {stop }}<\frac{\dot{\varphi}}{2 \pi}
$$

This means that there is a minimal speed of the gearbox the filter bank cannot operate below. Therefore it would seem that the stopband frequency should be as low as possible.

However, a high selectivity comes at a cost. In transient operation, e.g. a run-up of the gearbox the weights are not constant, but change slowly. If the selectivity of the filter is chosen too high, it will also reject fluctuation of the weights. This will degrade performance significantly. Therefore, at given minimum and maximum speed of the gearbox there is an optimal stopband frequency. On the measured data of gearbox A a stop band frequency of $f_{\text {stop }}=22 \mathrm{~Hz}$ and an attenuation of $84 \mathrm{~dB}$ yield optimal performance for a Chebyshev type II filter of order 4 .

\section{Vold-Kalman filtering}

The Vold-Kalman filter serves the same purpose of decomposing a signal into spectral components using a reference oscillator. It has initially been proposed in [9]. There are two distinct formulations of the algorithm. The first type calculates the decomposition of the signal $v$ into the spectral components $\tilde{v}_{k}$ in the time domain. The second type calculates the complex magnitude of the spectral components. This is equivalent to the determination of the Fourier coefficients $c_{k}$. It is generally agreed upon that the accuracy of Vold Kalman filters is very good. Therefore, researchers have tried to adapt the filter to real-time [10] or to make it on-line capable [11].

Because the Vold-Kalman filter calculates a globally optimal decomposition of the signal into its spectral components, the computational effort is higher than that of comparable methods. While [11] reports real-time capability on similar computer hardware as used in this paper, the 
Heterodyne filter
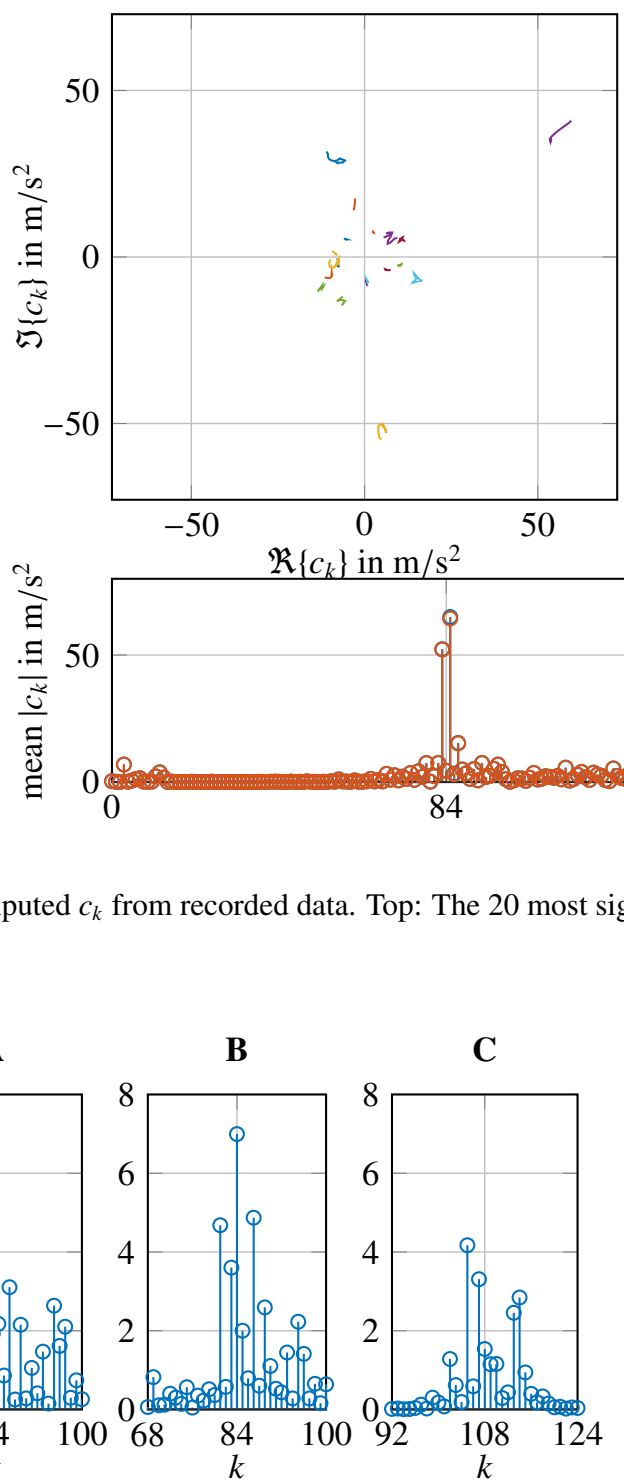

$\mathfrak{R}\left\{c_{k}\right\}$ in $\mathrm{m} / \mathrm{s}^{2}$
B

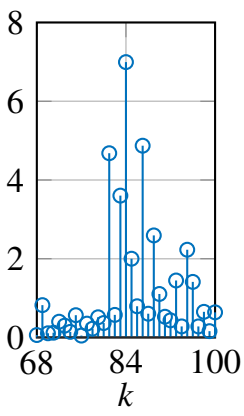

C

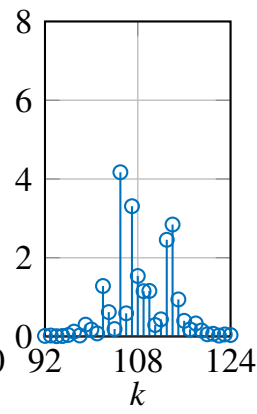

Integral transform

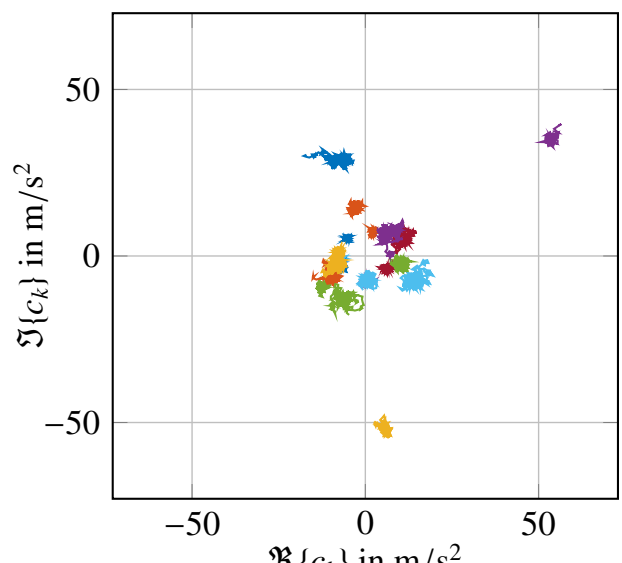

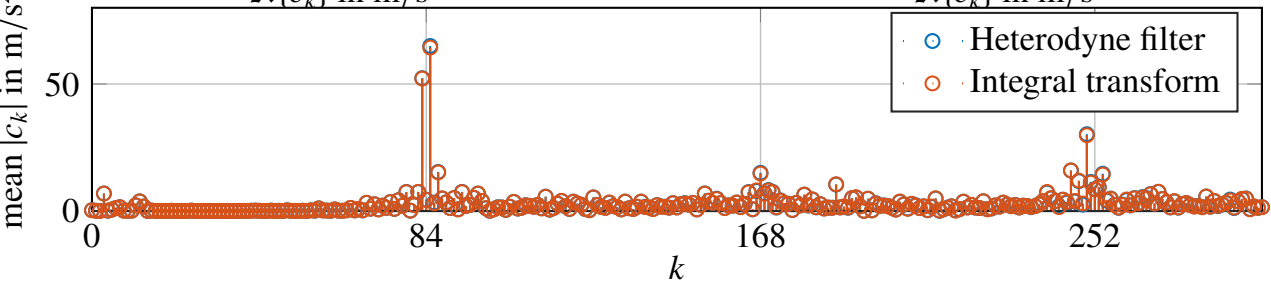

Figure 4. Computed $c_{k}$ from recorded data. Top: The 20 most significant; Bottom: Mean 0 to $3.5 Z$.

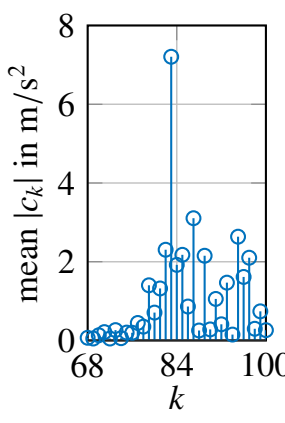

Figure 5. Input speed: $7000 \mathrm{~min}^{-1}$, load: $10 \mathrm{~N} \mathrm{~m}$.

conditions are less demanding than in the case of high speed planetary gearboxes. There, a sampling rate of $2048 \mathrm{~Hz}$ is used while in this study a sampling frequency of $80 \mathrm{kHz}$ is required. Also, only five orders are tracked in [11]. In addition the algorithm is executed in a block-wise fashion leading to a delay of up to $146 \mathrm{~ms}$. An other investigation [10] reports that it takes at least $21.7 \mathrm{~s}$ to track four orders in a $5 \mathrm{~s}$ signal at a sampling rate of $2 \mathrm{kHz}$.

These computational properties may be acceptable for condition monitoring, but are very problematic for active vibration control. Incorporation of the VKF into an AVC algorithm seems unlikely in this high speed application. Therefore, it will be not investigated further.

\section{Results}

There are two performance measures for a real-time order tracking algorithm: Accuracy and execution time. Figure 4

depicts the results of both the heterodyne filter and the integral transform. The vibration of gearbox A running at an input speed of $10000 \mathrm{~min}^{-1}$ and an output load of $30 \mathrm{Nm}$ has been recorded for a time of $0.8 \mathrm{~s}$. From this data, the Fourier coefficients $c_{k}$ are computed. The most significant coefficients are in the vicinity of the number of teeth on the ring gear $Z$. This is expected from Section 3 and Equation (3). Also, the nonlinear dynamics of the gear meshing excite higher gear mesh order around $2 Z$ and $3 Z$.

The results of both approaches are very similar. The integral transform yields a greater fluctuation of the coefficients than the heterodyne filter bank. The mean values of the computed coefficients are nearly identical. Therefore it has to be concluded that there is no significant difference in accuracy between the two approaches. Both approaches are then tested in terms of execution time on the test rig. The number of coefficients to be extracted is increased until CPU overload occurs. The heterodyne filter bank is able to extract the 100 most significant spectral components in real-time. The integral transform can track 20 orders. However, the program code has not been optimized and an improvement to the level of the heterodyne filter bank seems possible. In practice, it may be advisable to leave out some spectral components. If a certain component does not contribute to the total power of the signal, unit dedicated to its extraction will only yield noise. In this application the best reconstruction of the signal is achieved using 34 orders. Therefore, neither approach has a significant advantage in terms of execution speed.

Figure 5 compares the results of all three gearboxes during equal operating conditions. The load has been set to the maximum of gearbox B while the speed is limited by $\mathrm{C}$. Only the vicinity of the gear mesh order is depicted because 


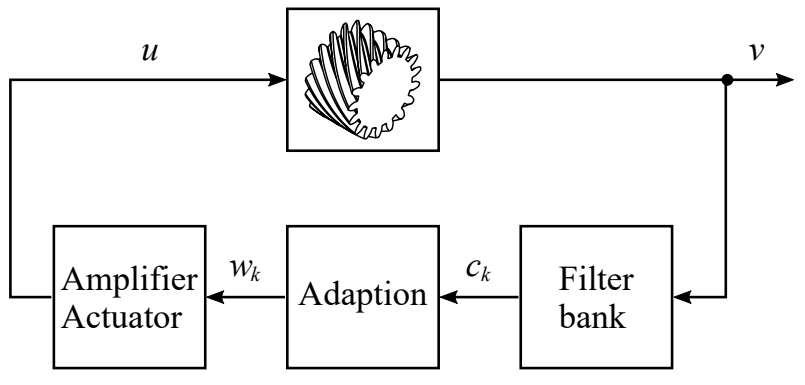

Figure 6. Architecture of the proposed AVC method.

here the most significant spectral component are located. The difference between spur (A,B) and helical (C) gears is to be expected. However, gearboxes A and B differ more than it would be expected from existing research. These two gearboxes share most constructive parameters (see Table 1). While the base frequency is mostly suppressed in A, it is most significant in B. This clearly contradicts existing research such as [1] or [3].

\section{Active Vibration Control}

Active vibration control of the gear meshing needs to utilize the specific structure of the excitation. Only this way sufficient performance may be achieved. One approach is to adapt the FxLMS algorithm to the planetary gearbox. It uses reference oscillators similar to those of the heterodyne filter bank. While it offers good performance, there is one drawback: The FxLMS algorithm relies on an accurate model of the secondary path. At frequencies of more than $4 \mathrm{kHz}$ small disturbances, even variations in temperature may change the frequency response of the secondary path considerably. Therefore, the performance of the FxLMS algorithm may degrade quickly. There have been remedies proposed for this problem. For example, Li et al [12] use on-line identification of secondary path. But this solution introduces new problems: The computational requirements will increase. Also the stability will depend on an accurate estimation of the secondary path, which is difficult to do outside of a laboratory.

An algorithm which does not rely on a model of the dynamics of the actuator or the structure will avoid this issue. Therefore, a new algorithm using real-time order tracking is proposed in Figure 6. From the measured vibration $v$ the Fourier coefficients $c_{k}$ are estimated using real-time order tracking. An optimization algorithm then chooses the output weights $w_{k}$. The output $u$ of the controller is determined by the complex weights $w_{k}$ and the reference oscillators.

$$
u=\sum_{k \in \mathcal{K}} w_{k} e^{i k \varphi}
$$

Only a relevant subset $\mathcal{K}$ is used for reasons discussed in Section 7. Because the performance measure is defined in terms of the Fourier coefficients, the problem will decompose into independent sub-problems. The objective is to minimize the magnitude of each coefficient $\left|c_{k}\right|$ individually by adjusting the corresponding output weight $\mathfrak{R}\left\{w_{k}\right\}$ and
$\mathfrak{J}\left\{w_{k}\right\}$. This leads to a much easier estimation of the gradient, because the dimension is reduced to 2. Also, the step size may be adjusted more efficiently because an estimation of the Hessian matrix is feasible. A discussion of an actual implementation would go beyond the scope of this paper.

\section{Conclusion}

Two methods of real-time order tracking have been developed: The heterodyne filter bank and the integral transform. Their respective accuracy and computational efficiency are both sufficient for the proposed high-speed real-time use. This has been validated using a test rig. The methods utilize the characteristic modulation of the vibration excited by a planetary gearbox. From existing research it it concluded that the Vold-Kalman filter does not meet the requirements on the computational effort. The architecture for an active vibration control algorithm using real-time order tracking has been proposed. In the next step, an actual implementation of AVC should be performed. Also the experimental data shows that the modulation in planetary gearboxes is more complex than commonly assumed. Therefore, a comparative study of different commercial gearboxes is planned.

\section{References}

[1] P. Mcfadden, J. Smith, Proceedings of the Institution of Mechanical Engineers, Part C: Journal of Mechanical Engineering Science 199, 65 (1985)

[2] P. Borghesani, P. Pennacchi, S. Chatterton, R. Ricci, Mechanical Systems and Signal Processing 44, 118 (2014)

[3] J. McNames, Journal of Vibration and Acoustics 124, 150 (2001)

[4] D.F. Plöger, P. Zech, S. Rinderknecht, Experimental identification of high-frequency gear mesh vibrations in a planetary gearbox, in Proceedings of ISMA2016 International Conference on Noise and Vibration Engineering (Leuven, Belgium, 2016), pp. 911-924

[5] J.L. Hogan, Proceedings of the Institute of Radio Engineers 1, 75 (1913)

[6] L.C. Godara, Proceedings of the IEEE 87, 1975 (1999)

[7] M.A. Soderstrand, in Adaptive Filtering, edited by L. Garcia (InTech, 2011), pp. 359-398, ISBN 978953-307-158-9

[8] Y. Qu, D. He, J. Yoon, B. Van Hecke, E. Bechhoefer, J. Zhu, Sensors 14, 1372 (2014)

[9] H. Vold, J. Leuridan, SAE Technical Paper 931288, SAE International, Warrendale, PA (1993)

[10] M.C. Pan, C.X. Wu, Mechanical Systems and Signal Processing 21, 2957 (2007)

[11] M. Čala, P. Beneš, Implementation of the vold-kalman order tracking filters for online analysis, in 23rd International Congress on Sound and Vibration 2016 (ICSV 23) (2016), Vol. 1, pp. 367-374

[12] M. Li, T.C. Lim, W.S.S. Jr, Y.H. Guan, Smart Materials and Structures 14, 917 (2005) 